\title{
Advances in Diagnostic Assays for Tuberculosis
}

\author{
Stephen D. Lawn ${ }^{1,2}$ \\ ${ }^{1}$ Department of Clinical Research, Faculty of Infectious and Tropical Diseases, London School of Hygiene \\ and Tropical Medicine, London WC1E 7HT, United Kingdom \\ ${ }^{2}$ The Desmond Tutu HIV Centre, Institute for Infectious Disease and Molecular Medicine, Faculty of Health \\ Sciences, University of Cape Town, Cape Town 7925, South Africa \\ Correspondence: stephen.lawn@Ishtm.ac.uk
}

Approximately one-third of the global burden of tuberculosis (TB) remains undiagnosed each year and the vast majority of cases of multidrug-resistant TB remain undetected. Many countries still place heavy reliance on outdated technologies that are blunt and ineffective tools for controlling this epidemic. However, during the past 10 years, there has been substantial progress within the TB diagnostics developmental pipeline. Old technologies have been reviewed and improved and new technologies have been developed and evaluated and are now being implemented. This review summarizes these developments and describes the currently available diagnostic tools. Consideration is given to the requirements of future diagnostic tests and how these should be evaluated not only with regard to their diagnostic accuracy and operational feasibility, but ultimately in terms of whether they impact clinical outcomes cost effectively, especially for those most in need.

$\mathrm{T}_{\mathrm{n}}^{\mathrm{h}}$ he global burden of tuberculosis (TB) remains enormous, and the incidence rate is decreasing at just $2 \%$ per year (WHO 2013a). Although the reasons underlying this slow progress in TB control are complex, TB diagnostic tools have long been recognized as a critical weakness in the care pathway. It was estimated that of the 8.6 million cases that occurred in 2012, 3.0 million of these were missed by national notification systems. Moreover, just one in five of the 450,000 cases of multidrugresistant TB (MDR-TB) that occur worldwide each year was detected (WHO 2013a). To accelerate progress toward elimination of $\mathrm{TB}$ as a public health problem by 2050 , there is a need for major improvements in the diagnostic process and in the microbiological tools that are used (Abu-Raddad et al. 2009).

In low- and middle-income countries, which bear the vast majority of the global burden of disease, heavy reliance is still placed on outdated TB diagnostic tools such as direct sputum smear microscopy, solid culture, and chest radiography. These lack either sufficient sensitivity or specificity, are too slow, or are not available at the periphery of the health system where patients first seek care. Opportunities to intervene early in the disease are lost, and diagnosis based solely on clinical suspicion results in overprescription of TB treatment in some settings.

Whereas rapid and accurate point-of-care assays have greatly simplified the diagnosis of

Editors: Stefan H.E. Kaufmann, Eric J. Rubin, and Alimuddin Zumla

Additional Perspectives on Tuberculosis available at www.perspectivesinmedicine.org

Copyright (C) 2015 Cold Spring Harbor Laboratory Press; all rights reserved; doi: 10.1101/cshperspect.a017806

Cite this article as Cold Spring Harb Perspect Med 2015;5:a017806 
S.D. Lawn

blood-borne disease such as human immunodeficiency virus type 1 (HIV-1) infection and malaria, no comparable tools exist for TB. TB presents a far greater diagnostic challenge. Diagnostic tools must be able to detect pulmonary and extrapulmonary disease when testing a wide variety of clinical sample types, which may differ substantially in bacillary burden. The ability to reliably distinguish between active TB and latent infection is crucial. To reduce morbidity, mortality, and transmission risk, accurate diagnosis is needed in the early stages of disease when bacillary burden in samples may still be low. Assays should be useful in both adults and children as well as those with immunodeficiency due, for example, to HIV coinfection. The growing threat of drug resistance worldwide (WHO 2013a) means that drug susceptibility testing (DST) must be routinely performed, and the advantages of incorporating this within the initial diagnostic assessment are self-evident.

Appropriate diagnostic assays are required for use at all levels of the health-care system and the community, even in settings with the least developed infrastructure. Obtaining samples and testing them must be safe for the patient, for other patients, for the staff in the health-care environment, and for laboratory staff. Frequent failure of diagnosed TB cases to start TB treatment (MacPherson et al. 2014), often referred to as "initial treatment default," highlights the critical need for same-day diagnosis and treatment initiation. The complexity of these various requirements and the vastly differing settings in which the diagnostic assays need to be used means that it is highly unlikely that a single test will ever be developed, but rather it is likely that a range of different assays will be needed at different levels within the healthcare system.

The developmental pipeline for TB diagnostics has received much needed focus in recent years. Old technologies have been reviewed and improved and new ones have been developed, evaluated, and implemented. With a rapidly growing evidence base, the World Health Organization (WHO) has issued 11 separate policy statements between 2007 and 2014 regarding TB diagnosis and diagnostic tools (Table 1). Four of these address sputum smear microscopy. Two statements address culture-based systems for diagnosis and DST, and one addresses the use of line-probe assays (LPAs) for rapid molecular diagnosis of drug resistance. Negative recommendations have also been issued regarding the use of serodiagnostic assays and interferon- $\gamma$ release assays (IGRAs) for TB diagnosis. Following endorsement in December 2010, WHO issued a policy statement in 2011 on use of the Xpert MTB/RIF automated rapid molecular assay for diagnosis of pulmonary TB and detection of rifampicin resistance. Guidelines on Xpert MTB/RIF were updated in 2013 to incorporate recommendations on use in children and for diagnosis of extrapulmonary TB.

The inverse care law states that the availability of good medical care tends to vary inversely with the need for it in the population served (Hart 1971). This is very true of TB diagnostic capacity. The WHO regions with the greatest burden of TB (Africa and South and Southeast Asia) have the least capacity for TB culture and DST by phenotypic means or by line-probe assay, for example (Table 2). Even though a simple and effective means of improving smear microscopy using light-emitting diode (LED) microscopes was endorsed by WHO in 2011, uptake around the world is still at very low levels (Table 2). Thus, developments in new diagnostic tools and evolution of global policies must be accompanied by effective implementation, especially where the need is greatest.

\section{LABORATORY TECHNOLOGIES}

\section{Microscopy and Culture}

\section{Microscopy}

Light microscopy of sputum smears has been the mainstay of TB diagnosis for more than a century and remains the most common means of case detection with assessment of $>80$ million patients per year (Perkins 2009). It uses cheap equipment and materials and is relatively rapid. However, it is insensitive and requires significant effort on the part of the laboratory technologist who has to meticulously examine dozens of microscopic fields such that quality 
Advances in Diagnostic Assays for Tuberculosis

Table 1. World Health Organization (WHO) policy statements on tuberculosis diagnosis (2007-2013)

\begin{tabular}{|c|c|c|c|}
\hline Year & Policy & Summary of recommendation & Reference \\
\hline 2007 & $\begin{array}{l}\text { Definition of a new smear- } \\
\text { positive TB case }\end{array}$ & $\begin{array}{c}\text { Can be based on the presence of } \geq 1 \text { acid-fast bacilli in } \\
\geq 1 \text { sputum sample in quality-assured laboratories }\end{array}$ & WHO 2007a \\
\hline 2007 & $\begin{array}{l}\text { Number of smears for the } \\
\text { diagnosis of pulmonary TB }\end{array}$ & $\begin{array}{l}\text { Number of samples tested can be reduced from three to } \\
\text { two in quality-assured laboratories }\end{array}$ & WHO 2007b \\
\hline 2007 & $\begin{array}{l}\text { Use of liquid culture medium } \\
\text { for culture and DST }\end{array}$ & $\begin{array}{l}\text { Use of liquid medium for culture, rapid species } \\
\text { identification, and DST recommended in low- and } \\
\text { middle-income countries }\end{array}$ & WHO 2007c \\
\hline 2008 & Use of line probe assays & $\begin{array}{l}\text { Molecular line probe assays are recommended for rapid } \\
\text { screening for MDR-TB }\end{array}$ & WHO 2008 \\
\hline 2011 & $\begin{array}{l}\text { Same-day diagnosis of TB by } \\
\text { microscopy }\end{array}$ & $\begin{array}{l}\text { Recommends a case-finding strategy that examines two } \\
\text { consecutive specimens obtained on the same day }\end{array}$ & WHO 2011a \\
\hline 2011 & $\begin{array}{l}\text { Noncommercial culture and } \\
\text { DST methods to detect } \\
\text { MDR-TB }\end{array}$ & $\begin{array}{l}\text { Microscopic observation of drug susceptibility } \\
\text { (MODS), nitrate reductase assay (NRA), and } \\
\text { colorimetric redox indicator (CRI) are recommended } \\
\text { for use in reference laboratories }\end{array}$ & WHO $2011 b$ \\
\hline 2011 & $\begin{array}{l}\text { Fluorescent light-emitting } \\
\text { diode (LED) microscopy } \\
\text { for TB diagnosis }\end{array}$ & $\begin{array}{l}\text { Ziehl-Neelen light microscopy and conventional } \\
\text { fluorescence microscopy should be replaced by LED } \\
\text { microscopy }\end{array}$ & WHO 2011c \\
\hline 2011 & $\begin{array}{l}\text { Commercial serodiagnostic } \\
\text { tests for diagnosis of } \mathrm{TB}\end{array}$ & $\begin{array}{l}\text { Strong recommendation that existing commercial } \\
\text { serological tests for } \mathrm{TB} \text { are not used for diagnosis of } \\
\text { pulmonary or extrapulmonary } \mathrm{TB}\end{array}$ & WHO 2011d \\
\hline 2011 & $\begin{array}{l}\text { Use of interferon- } \gamma \text { release } \\
\text { assays (IGRAs) in low- and } \\
\text { middle-income countries }\end{array}$ & $\begin{array}{l}\text { IGRAs should not be used in low- and middle-income } \\
\text { countries for the diagnosis of pulmonary or } \\
\text { extrapulmonary TB or to replace tuberculin skin } \\
\text { testing for diagnosis of latent TB }\end{array}$ & WHO 2011e \\
\hline 2011 & $\begin{array}{l}\text { Xpert/RIF for rapid detection } \\
\text { of TB and rifampicin } \\
\text { resistance }\end{array}$ & $\begin{array}{l}\text { Xpert/RIF should be used as the initial diagnostic test in } \\
\text { individuals suspected of MDR-TB or HIV-associated } \\
\text { TB; Xpert/RIF may also be used as a follow-on test to } \\
\text { microscopy in settings in which HIV and MDR-TB } \\
\text { are a lesser concern }\end{array}$ & WHO $2011 \mathrm{f}$ \\
\hline 2013 & $\begin{array}{l}\text { Xpert MTB/RIF for the } \\
\text { diagnosis of pulmonary and } \\
\text { extrapulmonary TB in } \\
\text { adults and children: policy } \\
\text { update }\end{array}$ & $\begin{array}{l}\text { Xpert/RIF should be used as the initial diagnostic test in } \\
\text { adults and children suspected of MDR-TB or HIV- } \\
\text { associated TB; Xpert should be used as the preferred } \\
\text { diagnostic test for CSF samples in those with } \\
\text { suspected TB meningitis and may be used as a } \\
\text { replacement for testing lymph node and other tissues }\end{array}$ & WHO 2013b \\
\hline
\end{tabular}

DST, drug susceptibility testing; CSF, cerebrospinal fluid; MDR-TB, multidrug-resistant TB.

is very operator-dependent. The sensitivity of smear microscopy is limited to detection of samples with greater than 10,000 bacilli per milliliter of sputum. Bacillary concentrations in sputum are typically lower in patients with HIV coinfection, such that the sensitivity of smear microscopy is often $\sim 20 \%$ lower in these patients (Gupta et al. 2013). Maintaining functional equipment and proficient microscopists out to the periphery of the health-care system in resource-limited settings has proved challenging. The specificity of microscopy is generally good in high TB burden settings, but it is lower in high-income countries where the proportion of positive sputum smears that is attributable to nontuberculous mycobacterial species is greater.

Two methods have been developed to increase the sensitivity of smear microscopysputum processing before preparation of smears 
S.D. Lawn
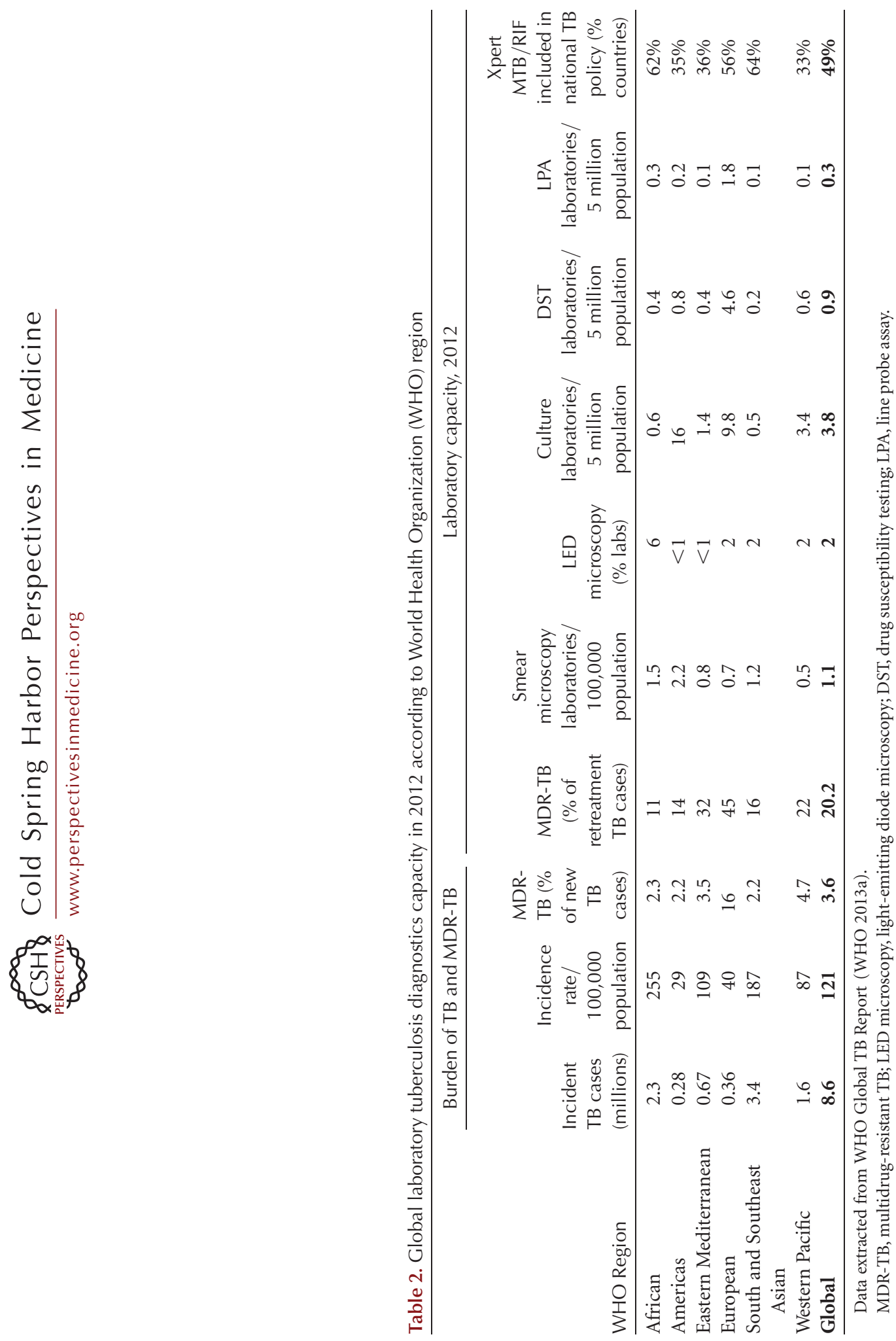
and use of fluorescent staining. Numbers of methods for sputum liquefaction (e.g., using inexpensive household bleach) and concentration (e.g., using centrifugation) have been shown to increase yield (Steingart et al. 2006a). Fluorescence microscopy for Mycobacterium tuberculosis was developed 70 years ago and allows slide examination with a medium power $(25 \times-$ $40 \times$ ) objective, increasing the speed of reading slides and increasing sensitivity by $\sim 10 \%$ with no loss in specificity (Steingart et al. 2006b). A major barrier to use of fluorescence microscopy in resource-limited settings was the high equipment cost and short bulb life. However, this problem has now been overcome by the development of microscopes with ultrabright lightemitting diodes (LEDs), which have low energy requirements (allowing battery operation) and a life span of $>10,000 \mathrm{~h}$ (Perkins 2009; Minion et al. 2011a). High-performance and inexpensive LED microscopes are now available and are increasingly being used, although the rate of scale-up is slow (Table 2). Development of automated microscopy has resulted in prototype devices, but successful devices have not yet emerged.

The Xpert MTB/RIF has now been implemented nationwide as a replacement for smear microscopy in South Africa, and it is possible that other high-burden countries may also do so in the future. However, paradoxically, smear microscopy is unlikely at present to be replaced as a primary diagnostic screening tool in industrialized countries where detection of nontuberculous mycobacterial species is of greater importance than in resource-limited settings.

\section{Culture-Based Diagnosis Drug Susceptibility Testing}

The most sensitive means of detecting $M$. tuberculosis is the culture of processed samples on enriched media. If culture is performed in high-burden countries, this usually uses solid egg-based media (Lowenstein-Jensen or Ogawa) with subsequent visualization of colonies. This requires $6-8$ wk to rule out growth because M. tuberculosis replicates very slowly. Use of selective liquid media and growth indicator sys- tems increase both the sensitivity and speed of detection. Time to detection is shortest using liquid culture with continuous automated monitoring systems, approximately halving the time to detection compared with culture on solid media (Perkins 2009). The emergence of the global epidemic of drug-resistant TB worldwide during the past decade has emphasized the need for much wider availability of culture for both diagnosis and DST. The most widely used method in resource-limited settings has been indirect DST of positive cultures on solid media, typically taking 2-3 mo. Incorporation of DST into automated liquid culture detection can reduce time to culture and DST results to $\sim 2 \mathrm{wk}$. In 2007, WHO endorsed the use of liquid culture media and phenotypic DST in low- and middle-income countries (Table 1).

As liquid culture is highly sensitive for growth of a wide range of nontuberculous mycobacteria, including environmental species, rapid and simple species identification is essential. Thus, WHO endorsement of liquid culture also included rapid species identification in solid or liquid culture isolates using a simple, low-cost, lateral-flow test (Capilia TB-Neo, Tauns Laboratories Inc., Japan) that detects the MPT-64 protein specific to M. tuberculosis (Hillemann et al. 2005). This has great advantages in terms of speed, simplicity, and cost over previously used phenotypic or genotypic methods of speciation.

Commercially available liquid culture systems have capital and reagent costs that are prohibitive for many resource-limited countries. Moreover, both the specimen processing and culture systems are relatively complex procedures that are challenging to perform outside reference laboratories. Thus, although commercial liquid culture systems and line-probe assays emerged in 2008 as the WHO-recommended gold standard endorsed for rapid detection of MDR-TB (Table 1), their technical complexity, cost, and the requirement for sophisticated laboratory infrastructure have limited their implementation (Table 2). In view of this, several other noncommercial, low-cost methods of culture and DST that require less sophisticated laboratory infrastructure have also been developed. 
S.D. Lawn

In 2011, WHO endorsed the use of three of these as an interim solution while capacity for more technically demanding assays was being developed (WHO 2011b). These assays included the microscopic observation of the drug susceptibility (MODS) assay, the nitrate reductase assay (NRA), and the colorimetric redox indicator (CRI) assay. All three rely on detection of growth of M. tuberculosis in drug-free and drugcontaining media. MODS is a microcolony liquid culture method conducted in 24-well culture plates with microscopic detection of early growth with $M$. tuberculosis through recognition of highly characteristic cord formation using an inverted microscope (Moore et al. 2006). Inclusion of additional TB drug-containing wells allows evaluation of susceptibility to a range of additional drugs. MODS has high accuracy for diagnosis of MDR-TB and provides a result within a mean turnaround time of $10 \mathrm{~d}$ (Minion et al. 2010).

The nitrate reductase phenotypic assay is a colorimetric method, which can be used as a direct method on sputum or as an indirect method on cultures (Musa et al. 2005). The assay is based on the ability of M. tuberculosis to reduce nitrate, which is detected by a color reaction on solid media. The CRI assay is based on the reduction of a colored indicator added to culture medium in a microtiter plate after exposure of $M$. tuberculosis to anti-TB drugs (Palomino et al. 2007). A simple visual readout is therefore possible.

WHO did not find sufficient evidence in 2011 to endorse the thin-layer agar (TLA) or phage-based assays. The TLA assay works on the same basis as MODS, but it uses drug-free and drug-containing agar with a colorimetric indicator in segmented Petri dishes (Robledo et al. 2008). Phage-based assays rely on mycobacteriophages, which infect $M$. tuberculosis bacilli if they are viable. Thus, detection of replicating bacteriophages in decontaminated specimens in the presence or absence of drug provides a rapid means of assessing drug susceptibility (Kiraz et al. 2007). A commercially available version of this assay (FASTPlaqueTB, Biotec, UK) uses a conventional plaque readout on a lawn of rapidly growing mycobacterial on solid media, giving results within $48 \mathrm{~h}$.
Field experience and operational research are providing an increasing evidence base for these assays as an alternative to commercial liquid culture and DST. Despite comparatively low costs, they remain relatively labor-intensive, requiring preinoculation processing and repeated examinations during incubation. These assays are not as yet being widely implemented.

\section{Nucleic Acid Amplification Tests}

Major progress has been made in the rapid molecular detection of $M$. tuberculosis using nucleic acid amplification tests (NAATs). These assays use a range of molecular techniques including polymerase chain reaction (PCR), realtime PCR, isothermal amplification, strain displacement or transcription-mediated amplification, and ligase chain reaction (Drobniewski et al. 2013). These are often combined with highly specific detection systems with hybridization with specific oligonucleotide probes to increased assay specificity. Speed and biosafety are the main advantages as containment is only required for the initial sample processing.

The first generation of assays developed 20 years ago showed excellent performance in terms of speed and specificity. However, although analytic performance was high in laboratory-based evaluations and in studies of smear-positive clinical samples, sensitivity for smear-negative samples was limited, potentially being compromised by the presence of PCR inhibitors or by loss of nucleic acids during sample processing. These initial assays were therefore not widely implemented as they were unable to compete with culture with regard to either sensitivity or DST, and their cost and complexity resulted in their use being largely restricted to high-income countries (Perkins 2009). However, development of newer assays and the growing need for rapid molecular detection of drugresistant TB have resulted in $\mathrm{WHO}$ endorsement of rapid molecular assays in recent years, including line-probe assays and the Xpert MTB/RIF assay (Table 1 ). Table 3 shows the range of NAATs that were commercially available in 2013 for diagnosis of pulmonary TB directly from sputum samples. These include assays launched 20 years ago to assays only recently marketed. 

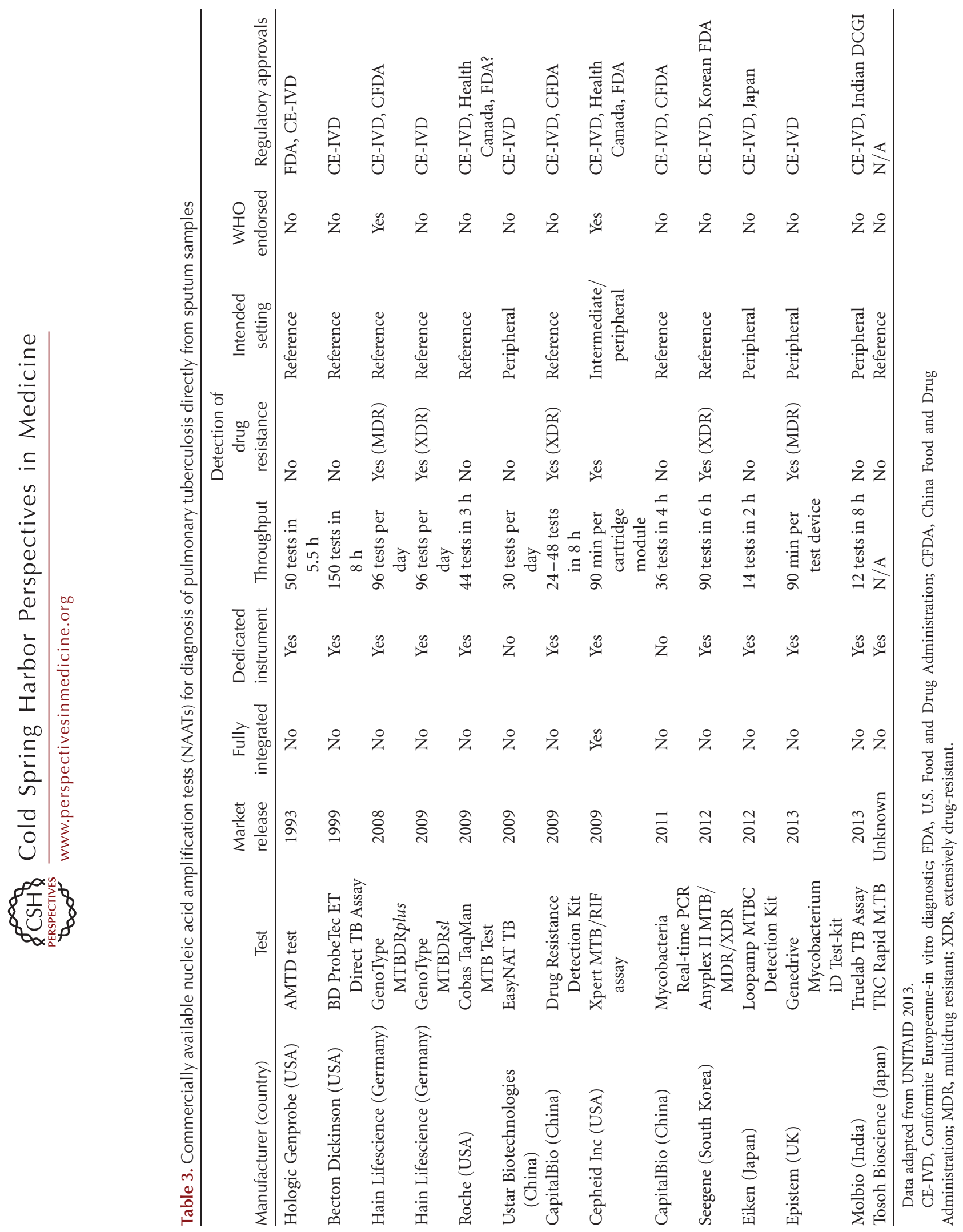
S.D. Lawn

\section{Line Probe Assays}

Line probe assays (LPAs) use PCR amplification of DNA sequences that are M. tuberculosis-specific and that are sites of mutations that are known to confer drug resistance. Detection uses DNA probes bound to solid membranes and hybridization is detected colorimetrically, producing visible bands that indicate the presence of $M$. tuberculosis and either a sensitive or resistant genotype.

To enhance the capacity for rapid diagnosis of MDR-TB, WHO in 2008 approved the use of LPAs for the rapid molecular detection of drug resistance in smear-positive specimens or culture isolates (WHO 2008). The main commercially available LPAs are the INNO-LiPA Rif.TB assay (Ommunogenetics, Zwijndrecht, Belgium) and, providing detection of both rifampicin and isoniazid resistance mutations, the GenoType MTBDR and MTBDRplus assays (Hain LifeScience GmbH, Nehren, Germany) (Morgan et al. 2005; Ling et al. 2008). More recently, the newer MTBDRplus version 2.0 assay (Hain Lifescience) has shown improved performance, with sensitivity for sputum smearnegative TB that is similar to that of the Xpert MTB/RIF assay (Barnard et al. 2012; Crudu et al. 2012).

In 2009, the Genotype MTBDRsl assay (Hain Lifescience) was released, providing capacity for expanded DST to include second-line drugs (Hillemann et al. 2009). WHO expert review of data concerning this assay in 2012 found moderate test sensitivity for the detection of fluoroquinolone and second-line injectable resistance with high specificity. It was concluded that although the Genotype MTBDRsl assay cannot be used as a replacement for conventional phenotypic DST, it does provide a rapid rulein (but not rule-out) screen for XDR-TB (WHO 2013c). However, because cross-resistance between second-line injectables is incomplete, the assay cannot be used to identify individual drugs to be used for treatment.

\section{Xpert MTB/RIF Assay}

GeneXpert (Cepheid Inc, Sunnyvale, CA, USA) is a self-contained, fully integrated and auto- mated cartridge-based diagnostic platform that can be operated with minimal technical expertise (Lawn et al. 2013). The Xpert MTB/RIF cartridges incorporate microfluidics technology and fully automated nucleic acid analysis to purify, concentrate, detect, and identify targeted nucleic acid sequences from unprocessed clinical samples using real-time PCR technology and a molecular beacon detection system (Fig. 1). This permits identification of the M. tuberculosis complex within $2 \mathrm{~h}$ and simultaneous detection of the presence or absence of mutations that confer rifampicin resistance in the 81-base-pair core region of the $r p o B$ gene (Lawn and Nicol 2011). Following initial endorsement in December 2010, WHO issued policy recommendations in 2011 for the use of this assay for diagnosis of pulmonary TB (WHO 2010), and these were updated in 2013 to include diagnosis of TB in children and diagnosis of extrapulmonary TB (WHO 2013b).

Extensive data from around the world are available on the diagnostic accuracy of the Xpert MTB/RIF assay for pulmonary TB (Boehme et al. 2010). Pooled summary estimates of sensitivity for sputum-culture-positive disease show that Xpert MTB/RIF detects approximately nine out of 10 cases with a pooled specificity of $99 \%$ (95\%CI, 98\%-99\%) including almost all smear-positive cases and approximately twothirds of smear-negative cases (Table 4). Despite the high frequency of smear-negative disease, the sensitivity of Xpert MTB/RIF in those with HIV-coinfection was not substantially lower than that in HIV-negative participants (Steingart et al. 2014). Accumulating evidence now supports the role of the Xpert MTB/RIF assay for diagnosis of some forms of extrapulmonary TB (Denkinger et al. 2014). Sensitivity varies according to sample type, whereas specificity is uniformly high across all types. Useful sensitivity is observed when testing biopsies and needle aspirates of lymph nodes, biopsies of other tissues, and cerebrospinal fluid samples (Table 4). However, sensitivity is much more limited when testing pleural fluid samples, leading to $\mathrm{WHO}$ recommendations that the assay can be used for diagnosis of $\mathrm{TB}$ meningitis and $\mathrm{TB}$ in lymph nodes and other tissues (WHO 2013b). 


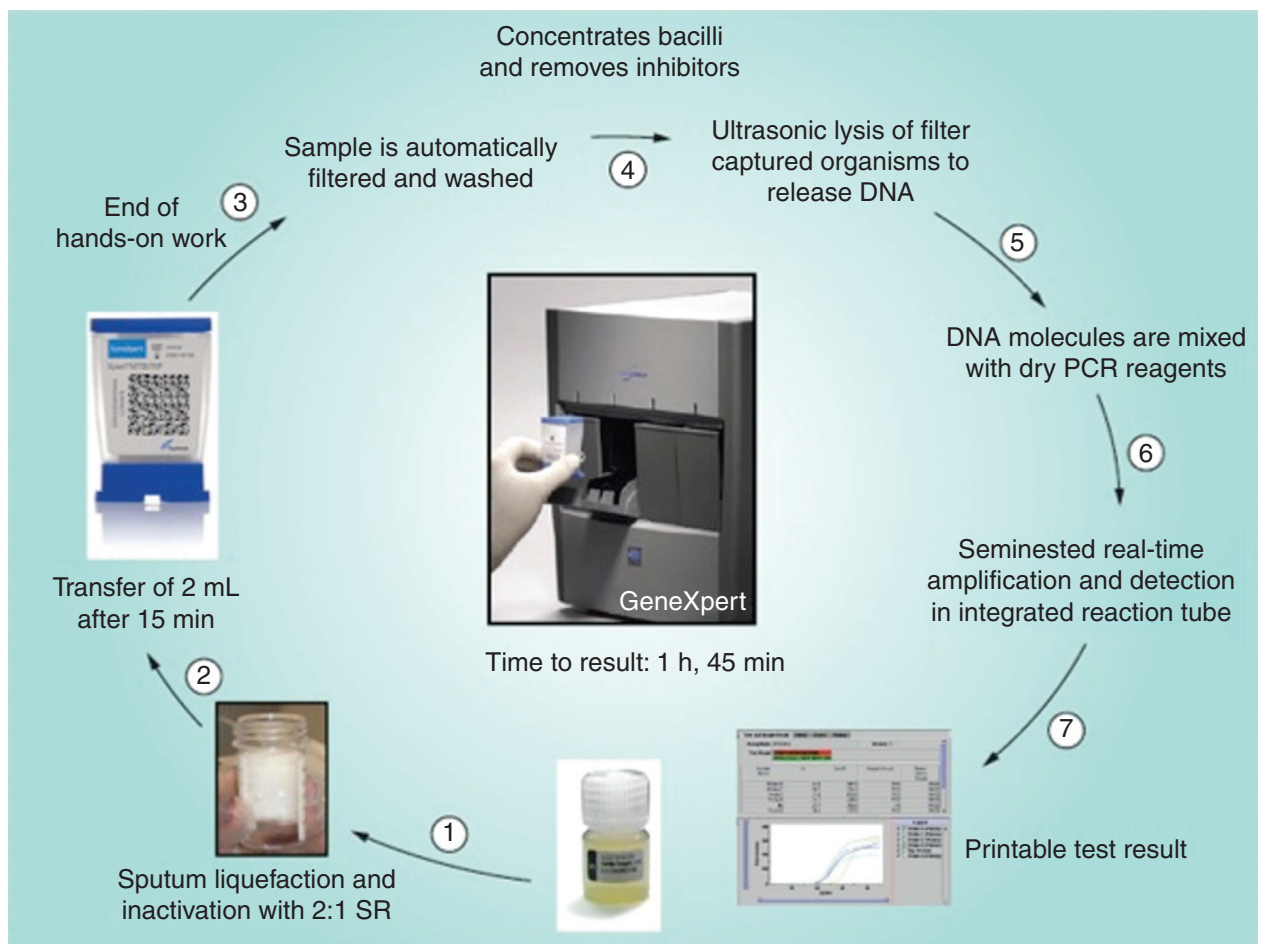

Figure 1. Schematic showing the sequential steps of the Xpert MTB/RIF assay test procedure. SR, sample reagent; PCR, polymerase chain reaction. (Figure reproduced from Lawn and Nicol 2011, with permission from the authors.)

The pooled sensitivity of Xpert MTB/RIF for detection of rifampicin resistance in pulmonary specimens was 95\% (Steingart et al. 2014), reflecting the proportion of rifampicin resistance that is mediated by mutations in the target region of the $r p o B$ gene. Pooled specificity for rifampicin resistance was suboptimal (98\%; 97\%-99\%), however, which means that in clinical populations with low prevalence of rifampicin-resistant $\mathrm{TB}$, the positive predictive value

Table 4. Pooled summary estimates of the sensitivity of the Xpert MTB/RIF assay for pulmonary and extrapulmonary tuberculosis (TB) compared with a culture reference standard

\begin{tabular}{ll}
\hline Sample types & Pooled sensitivity \\
\hline Respiratory specimens & \\
Overall & $89 \%(85 \%-92 \%)$ \\
Smear-positive & $98 \%(97 \%-99 \%)$ \\
Smear-negative & $67 \%(60 \%-74 \%)$ \\
Pulmonary TB in HIV-negatives & $86 \%(76 \%-92 \%)$ \\
Pulmonary TB in HIV-positives & $79 \%(70 \%-86 \%)$ \\
Nonrespiratory specimens & \\
Lymph node biopsies or aspirates & $83 \%(71 \%-91 \%)$ \\
Cerebrospinal fluid & $81 \%(59 \%-92 \%)$ \\
Pleural fluid & $46 \%(26 \%-68 \%)$ \\
\hline
\end{tabular}

Data summarized from Steingart et al. 2014 and Denkinger et al. 2014. 
of rifampicin-resistant result from Xpert MTB/ RIF is limited. Following modification of the assay cartridge and platform software in December 2011, however, data on the performance of the revised G4 version of the cartridge indicate higher specificity for rifampicin resistance (Osman et al. 2014).

The Xpert MTB/RIF assay is now being widely implemented around the world and, in 2011, South Africa took the bold decision to implement this nationwide as a replacement for sputum smear microscopy. Although the Xpert MTB/RIF assay is a substantial advance, it is an imperfect solution. High cost, sophisticated hardware, linkage to a computer, the need for an uninterrupted power supply, annual calibration, module breakdowns, and operator training requirements are all disadvantages in resource-limited settings (Trebucq et al. 2011). Although the assay can be completed within $2 \mathrm{~h}$, same-day diagnosis and treatment initiation are logistically challenging to achieve within overcrowded clinics in resource-limited settings. Economic and logistical issues mean that implementation of this assay will largely be constrained to the laboratory environment and away from the actual clinical interface.

\section{Developmental Automated Rapid Molecular Systems}

Fully automated systems that use isothermal amplification at lower operational temperatures have much lower power requirements than the Xpert MTB/RIF assay (Niemz and Boyle 2012; UNITAID 2013). Hand-held battery-powered systems the size of a smart-phone produce PCR product far more rapidly $(<30 \mathrm{~min})$ than GeneXpert and therefore may be much more readily used at the point-of-care. A number of new NAATs have been commercially launched, including some that are intended for use in peripheral laboratories (Table 3). It is anticipated that in the coming few years, a number of fully automated multiplexed assays (referred to as "fast-followers") with fully integrated sample processing will emerge to compete with the Xpert MTB/RIF assay, enabling both rapid TB diagnosis and detection of resistance to multiple drugs.

\section{Manual Loop-Mediated Isothermal Amplification Test}

Work continues on the development and evaluation of a simplified manual molecular assay for laboratory-based use in resource-limited settings, using loop-mediated isothermal amplification (LAMP) with a simple visual colorimetric readout (George et al. 2011; Mitarai et al. 2011). Rather than requiring repeated heating and cooling cycles used in PCR, such assays can be performed using a simple water bath, making the assay far more amenable to use within resource-limited settings. Expert review by WHO in 2013 of the Loopamp assay (Eiken Chemical Co., Japan) found that this could detect the vast majority of smear-positive samples and approximately half of smear-negative samples (WHO 2013d). However, specificity was insufficient $(<95 \%)$ for the assay to be recommended as a replacement for smear microscopy and further developmental work is needed, especially to improve specificity.

\section{Antigen Detection}

M. tuberculosis antigen detection has long been viewed positively as an option for TB diagnosis as this has the advantage of reflecting mycobacterial burden (active disease rather than latent infection) and not depending on the immune status of the individual. Moreover, analysis of urine rather than sputum samples is a very attractive option as this is simple to collect without generating hazardous bioaerosols, it is safe to handle in the laboratory, it has relatively few bacterial contaminants, and sample quality is unlikely to be highly variable (Peter et al. 2010).

A number of mycobacterial antigens can be detected in the urine of patients with pulmonary TB (Kashino et al. 2008), but the most promising of these to emerge is the cell wall lipopolysaccharide lipoarabinomannan (LAM) (Peter et al. 2010; Lawn 2012). A polyclonal antibody, sandwich enzyme-linked immunosorbent assay (ELISA) for urine LAM has been commercially 


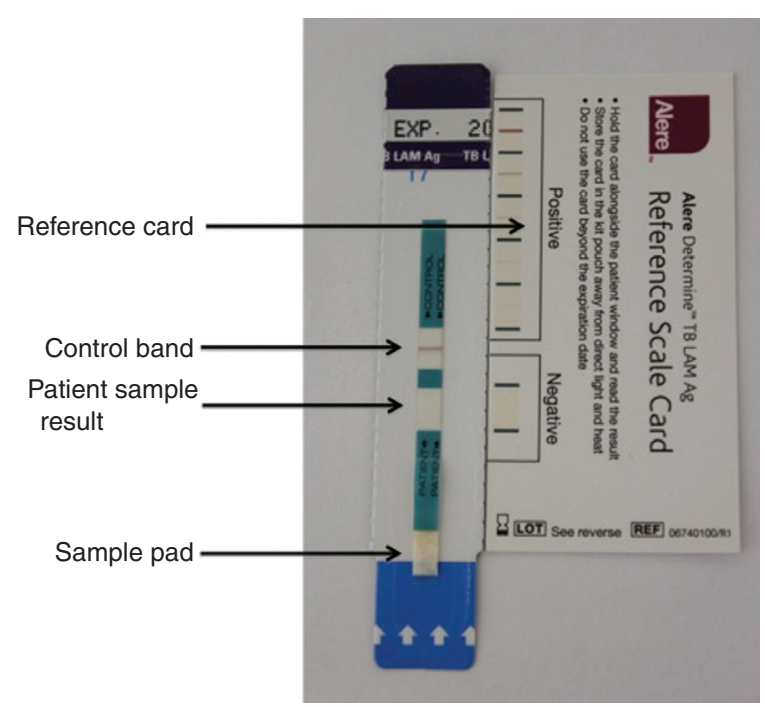

Figure 2. Photograph of a Determine TB-LAM Ag lateral flow test strip. Urine $(60 \mu \mathrm{L})$ is applied to the sample pad, and after 25-35 min of incubation at room temperature, the control band is checked and the sample test result is read with comparison to the reference card. The presence of a band of sufficient intensity in the sample window indicates the presence of lipoarabinomannan (LAM) in the urine, providing a rapid diagnosis of tuberculosis. (Figure reproduced from Lawn 2012, with permission from the author.)

available for a number of years (Minion et al. 2011b), and more recently, a simple, low-cost, lateral flow version of this assay has been developed and this can be used at the point-of-care (Fig. 2) (Lawn et al. 2012a; Peter et al. 2012).

Multiple studies have shown that the specific niche for this assay is for diagnosis of HIVassociated TB in patients with advanced immunodeficiency (CD4 cell counts $<200$ cells $/ \mu \mathrm{L}$ ), such as those being screened in antiretroviral treatment clinics or HIV-infected medical inpatients. Studies from South Africa have reported that among those with the lowest CD4 cell counts, the assay can diagnose between one-half and two-thirds of cases with high specificity within $30 \mathrm{~min}$. Those detected by this assay are likely to have disseminated TB, and growing evidence suggests that the main mechanism underlying the presence of LAM in urine is actual renal involvement with TB following hematogenous seeding (Lawn 2012). This explains why the utility of the assay is restricted to those with advanced immunodeficiency, poor prognostic characteristics, and high mortality risk (Lawn et al. 2012b; Talbot et al. 2012). These are the very patients in whom TB diagnosis and initia- tion of treatment are most urgent. Studies of the clinical impact of using this assay are awaited. The growing evidence base on this assay is being reviewed by WHO in 2014.

\section{Serological Assays}

Serological assays have long been attractive as a prospect for a simple, rapid, low-cost, and noninstrumented assay that uses a finger prick blood sample and can be used in the very lowest tier of the health-care system and community. However, systematic reviews of a large range of commercially available serodiagnostic assays for TB used widely in some parts of the world revealed that they had very limited accuracy and were of no clinical value (Steingart et al. 2007a,b). In 2011, WHO took the unprecedented step of issuing a negative recommendation against the use of currently available assays (WHO 2011d). However, future use of serodiagnostic assays has not been discounted and active research and development are ongoing.

A better understanding of the complex and evolving nature of the humoral immune response during the different stages of infection 
S.D. Lawn

and disease is needed. The multiplicity of different $M$. tuberculosis exposure, infection, and disease states (Lawn et al. 2011; Young et al. 2009) is likely to be associated with overlapping humoral immune response profiles, such that limited specificity is likely to remain the Achilles heel in high-burden settings. Early tests were based on crude mixtures of antigens that lacked specificity, but now an expanding range of purified immunodominant antigens has been identified (Steingart et al. 2009). Assays using individual antigens are unlikely to have sufficient sensitivity, and so it is likely that combinations of purified antigens will be needed. Sensitivity is usually limited in those with HIV coinfection, although serological responses to certain antigens appear to be preserved in this patient group (Yu et al. 2012). Serological assays have frequently been evaluated in small case-control studies in which highly selected study groups have been recruited, potentially giving a false impression of their accuracy. Much larger prospective studies are needed.

\section{Interferon- $\gamma$-Release Assays}

Interferon- $\gamma$-release assays (IGRAs) detect interferon- $\gamma$ production by CD4 lymphocytes in the blood of individuals in response to ex vivo stimulation with RD1-encoded M. tuberculosisspecific antigens, such as culture filtrate protein-10 (CFP-10) and early secretory antigen-6 (ESAT-6) (Pai et al. 2008). Positive responses indicate immune sensitization by prior exposure to the organism. However, because these assays lack sensitivity among those with active disease and cannot distinguish between active disease and latent infection, these assays should not be used in place of standard diagnostic methods for diagnosing active TB. WHO has specifically recommended that these assays not be used in low- or middle-income countries for diagnosis of active disease or latent infection (WHO 2011d). However, in limited clinical situations such as those in which all microbiological tests are negative and in diagnosis in children, IGRAs can contribute supplementary information as part of a diagnostic work-up (European Centre for Disease Control 2011).
However, this role is limited as the assays can neither be used to rule in nor rule out TB.

\section{Other Developmental Assays}

When M. tuberculosis is actively replicating, volatile organic compounds (VOCs) are released and are detectable in sputum, urine, and breath, thereby potentially forming the basis of a diagnostic assay (UNITAID 2012). As a proof of concept, Giant African Pouch rats can be trained to identify TB in sputum samples by olfaction, but this does not constitute a viable, scalable solution. Thus, a field of diagnostics research is exploring the use of "breathalyzers" and "electronic noses" that detect VOCs using chemical sensors and pattern recognition systems (Bruins et al. 2012; Phillips et al. 2012). The advantages of using such tests to screen for TB would be speed, noninvasiveness, and the lack of need for sample processing. However, to date, early developmental systems have required sophisticated laboratory equipment and highly skilled technicians and have lacked specificity (Phillips et al. 2012). Thus, viable prototypes for routine use have yet to emerge.

\section{DEVELOPING THE IDEAL DIAGNOSTIC TEST}

The ideal test for TB would be one that could be performed at the point-of-care, providing immediate results that can be used to reliably inform treatment decisions without the need for further laboratory-based testing. The test should be readily used at all levels of the health-care system, on hospital wards, outpatient clinics, peripheral health posts, mobile medical services, medical outreach teams visiting the remotest locations, and within the patient's home. Standalone diagnostic devices already exist for a range of infectious diseases, including HIV infection, malaria, hepatitis B, syphilis, and Chagas' disease. However, TB diagnosis presents a greater challenge. Unlike many of these diseases, TB is not primarily blood-borne, a wide spectrum of infection and disease states occur, and disease may be caused by very low bacillary numbers and may occur at any anatomic site. Although use of a single test for diagnosis would be ideal, an al- 
Advances in Diagnostic Assays for Tuberculosis

Table 5. Minimum specifications for a point-of-care diagnostic test for tuberculosis (TB)

\begin{tabular}{|c|c|}
\hline Parameter & Minimum specification required \\
\hline Outcome of testing & Initiation of treatment \\
\hline \multirow{3}{*}{$\begin{array}{l}\text { Sensitivity in adults (irrespective } \\
\text { of HIV status) }\end{array}$} & Smear-positive, culture-positive PTB: 95\% \\
\hline & Smear-negative, culture-positive PTB: $60 \%-80 \%^{a}$ \\
\hline & EPTB: detection preferred but not required \\
\hline Specificity in adults & Compared with culture: $95 \%$ \\
\hline \multirow{2}{*}{$\begin{array}{l}\text { Sensitivity in children } \\
\quad \text { (irrespective of HIV status) }\end{array}$} & Culture-confirmed PTB and EPTB: $80 \%$ \\
\hline & Probable PTB and EPTB: $60 \%$ \\
\hline \multirow[t]{2}{*}{ Specificity in children } & Compared with culture: $95 \%$ \\
\hline & In culture-negative probable cases: $90 \%$ \\
\hline Throughput & 20 tests per day by a single operator \\
\hline Waste disposal & Environmentally friendly disposal feasible \\
\hline Storage and stability & No cold chain required; stable at $30^{\circ} \mathrm{C}$ for $2 \mathrm{yr}$ \\
\hline Instrumentation & $\begin{array}{l}\text { Maintenance free, robust in tropical climates, acceptable replacement } \\
\text { cost, portable (e.g., in backpack), can be battery operated, shock } \\
\text { resistant }\end{array}$ \\
\hline Operation & Requires minimal instruction \\
\hline Cost & Less than $\$ 10$ per test \\
\hline
\end{tabular}

Data adapted from Lemaire and Casenghi 2010 and McNerney and Daley 2011.

Recommendations of an expert meeting facilitated by Medecins Sans Frontieres (MSF) Access Campaign, Treatment Action Group (TAG), and Partners in Health (PIH) and convened in March 2009.

PTB, pulmonary tuberculosis; EPTB, extrapulmonary tuberculosis.

${ }^{\mathrm{a}}$ No consensus reached on lower limit.

ternative approach would be serial testing using a high-sensitivity, low-specificity screening test followed by referral for a definitive test. However, in general, the more complex the screening algorithm, the weaker it is likely to be. If the screening test were simple and low-cost, this might nevertheless provide a suitable approach for screening in very remote communities with poor access to health services.

The specifications of the "ideal" diagnostic test for TB are a subject of debate (McNerney and Daley 2011). Although such a test may never be developed, prespecified desirable characteristics are important for guiding ongoing research and development. Table 5 shows the minimum test specifications that were identified during an expert meeting in 2009, including diagnostic accuracy, issues related to operational feasibility in a range of environments and cost (Lemaire and Casenghi 2010). The Xpert MTB/ RIF assay has made progress toward fulfilling some of these goals, including diagnostic accuracy requirements in adults and being made available at a heavily subsidized cost of $\$ 9.98$ per test in low- and middle-income countries.
Drawbacks of the Xpert MTB/RIF assay include the sophisticated diagnostic platform, which is costly, needs to be linked to a computer, is subject to breakdown of the modules, requires annual calibration, has high power requirements, and lacks portability (Lawn et al. 2013). The next-generation rapid molecular devices have much simpler instrumentation, greater speed, and low power requirements (UNITAID 2013) but their diagnostic accuracy remains to be defined. Although these evolving rapid molecular assays offer the prospects of much improved TB diagnosis, they still remain far from the simplicity of a lateral-flow HIV test. Thus, there remains a huge need for ongoing biomarker discovery and research and development of new diagnostic formats (Wallis et al. 2013).

\section{EVALUATIONS OF NEW DIAGNOSTIC TESTS FOR TB}

As with any other medical technology, new TB diagnostic assays require thorough evaluation before their implementation into routine practice. The methods and nomenclature for ap- 
S.D. Lawn

praising diagnostic accuracy date back to 1947 (Yerushalmy 1947), with $2 \times 2$ tables being used to derive the familiar indices of sensitivity, specificity, positive and negative predictive values, and likelihood ratios. The laboratory-based analytic performance is often first assessed using specimen banks of clinical samples. Thereafter, diagnostic accuracy studies are conducted in clinical populations, and the Standards for Reporting Diagnostic Accuracy (STARD) statement provides an important framework for the standardized reporting of such studies (Bossuyt et al. 2003). Diagnostic accuracy studies need to be conducted in a wide variety of clinical populations so as to encompass the true spectrum of disease and patients. Systematic reviews and meta-analyses are a critical next step in the synthesis of the findings of these studies, with careful review of the biases inherent in the studies included.

A critical component of diagnostic accuracy studies is a robust reference standard against which to compare the new assay. This is particularly challenging for certain forms of extrapulmonary TB and especially for pediatric TB in which only a limited proportion of disease may be microbiologically proven. There is additional difficulty in which the reference standard and index test are performed on samples from different anatomic compartments. For example, many of the evaluations of the Determine TB-LAM Ag urine LAM assay have used sputum culture as the reference standard despite the fact that a high proportion of true-positive LAM tests occur in patients with either negative sputum tests or no sputum production (Lawn et al. 2014).

Diagnostic accuracy is, however, only one component in the evaluation of diagnostic assays and much else needs to be considered. Factors include patient acceptability, the logistics of the testing procedure, the safety of patients and health-care workers, the precise role of the test within the diagnostic algorithm, the impact of the test on clinical decision-making, impact on health outcomes, cost-effectiveness, and overall cost of implementation. Testing procedures must be patient friendly and take into account the indirect costs that patients often have to bear when making repeated attendances at health clinics. The speed and throughput of the assay, as well as its ease of use within the clinical or laboratory environment, are critical. Biosafety is a vital consideration both during sample collection and running the test.

The final goal of a new diagnostic test is not simply to achieve accurate diagnosis of TB but is rather to achieve a clinical impact (Bossuyt et al. 2012). At the level of the individual patient, the aim is to reduce TB-related morbidity and mortality and, at the community-level, the aim is to reduce TB transmission and the burden of TB. New diagnostic tests have to be evaluated within the context and complexities of the clinical care pathway. In the face of limited sensitivity of TB diagnostic assays, the decision for health-care workers to start or not start TB treatment is not straightforward but rather takes into account a complex array of information in addition to the results of diagnostic tests. Thus, new TB diagnostic tests need to be evaluated in pragmatic randomized trials to assess clinical impact (Dowdy et al. 2012). To take into account the full diagnostic process, these often use a cluster-randomized design, although in certain circumstances, individually randomized trials are the only viable option. Study outcomes may include the diagnostic process (e.g., proportion diagnosed, proportion treated, and time to initiation of treatment) and clinical outcomes (e.g., outcomes of TB treatment and survival). Although assessment of individual outcomes may be achievable, the secondary effects of early TB diagnosis resulting in reduced TB transmission and reduced long-term burden of TB in the community are much more difficult to quantify. Careful context-specific economic analyses are also needed to evaluate cost-effectiveness. In addition, because cost-effectiveness does not denote affordability, budget impact analyses are also needed. Only then can rational choices be made by national Ministries of Health and national TB control program.

\section{CONCLUSIONS}

Huge progress has been made in the TB diagnostic developmental pipeline during the past 10 years, resulting in steady evolution of WHO 
policies for TB diagnosis and drug susceptibility testing. Despite this encouraging progress, we remain very far from the much-needed test that allows rapid and accurate diagnosis in unselected patients at the point-of-care, resulting in improved clinical outcomes and reduced TB transmission. Although the current array of technologies that are emerging from the developmental pipeline need to be implemented as effectively as possible, there remains a huge need for ongoing biomarker discovery combined with development of innovative technologies that lead to new and effective tools.

\section{REFERENCES}

Abu-Raddad LJ, Sabatelli L, Achterberg JT, Sugimoto JD, Longini IM Jr, Dye C, Halloran ME. 2009. Epidemiological benefits of more-effective tuberculosis vaccines, drugs, and diagnostics. Proc Natl Acad Sci 106: 13980-13985.

Barnard M, Gey van Pittius NC, van Helden PD, Bosman M, Coetzee G, Warren RM. 2012. The diagnostic performance of the genotype MTBDRplus version 2 line probe assay is equivalent to that of the Xpert MTB/RIF assay. $J$ Clin Microbiol 50: 3712-3716.

Boehme CC, Nabeta P, Hillemann D, Nicol MP, Shenai S, Krapp F, Allen J, Tahirli R, Blakemore R, Rustomjee R, et al. 2010. Rapid molecular detection of tuberculosis and rifampin resistance. N Engl J Med 363: 1005-1015.

Bossuyt PM, Reitsma JB, Bruns DE, Gatsonis CA, Glasziou PP, Irwig LM, Moher D, Rennie D, de Vet HC, Lijmer JG. 2003. The STARD statement for reporting studies of diagnostic accuracy: Explanation and elaboration. Ann Intern Med 138: W1-W12.

Bossuyt PM, Reitsma JB, Linnet K, Moons KG. 2012. Beyond diagnostic accuracy: The clinical utility of diagnostic tests. Clin Chem 58: 1636-1643.

Bruins M, Rahim Z, Bos A, van de Sande WW, Endtz HP, van Belkum A. 2012. Diagnosis of active tuberculosis by enose analysis of exhaled air. Tuberculosis (Edinb) 93:232238.

Crudu V, Stratan E, Romancenco E, Allerheiligen V, Hillemann A, Moraru N. 2012. First evaluation of an improved assay for molecular genetic detection of tuberculosis as well as rifampin and isoniazid resistances. J Clin Microbiol 50: 1264-1269.

Denkinger CM, Schumacher SG, Boehme CC, Dendukuri N, Pai M, Steingart KR. 2014. Xpert MTB/RIF assay for the diagnosis of extrapulmonary tuberculosis: A systematic review and meta-analysis. Eur Respir J 44: 435-446.

Dowdy DW, Gounder CR, Corbett EL, Ngwira LG, Chaisson RE, Merritt MW. 2012. The ethics of testing a test: Randomized trials of the health impact of diagnostic tests for infectious diseases. Clin Infect Dis 55: 1522-1526.

Drobniewski F, Nikolayevskyy V, Maxeiner H, Balabanova Y, Casali N, Kontsevaya I, Ignatyeva O. 2013. Rapid diagnostics of tuberculosis and drug resistance in the indus-
Advances in Diagnostic Assays for Tuberculosis

trialized world: Clinical and public health benefits and barriers to implementation. BMC Med 11: 190.

European Centre for Disease Control. 2011. Use of interferon- $\gamma$ release assays in support of TB diagnosis. Stockholm: ECDC, Accessible at: http://ecdc.europa.eu/en/ publications/publications/1103_gui_igra.pdf.

George G, Mony P, Kenneth J. 2011. Comparison of the efficacies of loop-mediated isothermal amplification, fluorescence smear microscopy and culture for the diagnosis of tuberculosis. PLoS ONE 6: e21007.

Gupta RK, Lawn SD, Bekker LG, Caldwell J, Kaplan R, Wood R. 2013. Impact of human immunodeficiency virus and CD4 count on tuberculosis diagnosis: Analysis of citywide data from Cape Town, South Africa. Int J Tuberc Lung Dis 17: 1014-1022.

Hart JT. 1971. The inverse care law. Lancet 1: 405-412.

Hillemann D, Rusch-Gerdes S, Richter E. 2005. Application of the Capilia TB assay for culture confirmation of Mycobacterium tuberculosis complex isolates. Int J Tuberc Lung Dis 9: 1409-1411.

Hillemann D, Rusch-Gerdes S, Richter E. 2009. Feasibility of the GenoType MTBDRsl assay for fluoroquinolone, amikacin-capreomycin, and ethambutol resistance testing of Mycobacterium tuberculosis strains and clinical specimens. J Clin Microbiol 47: 1767-1772.

Kashino SS, Pollock N, Napolitano DR, Rodrigues V Jr, Campos-Neto A. 2008. Identification and characterization of Mycobacterium tuberculosis antigens in urine of patients with active pulmonary tuberculosis: An innovative and alternative approach of antigen discovery of useful microbial molecules. Clin Exp Immunol 153: 56-62.

Kiraz N, Et L, Akgun Y, Kasifoglu N, Kiremitci A. 2007. Rapid detection of Mycobacterium tuberculosis from sputum specimens using the FASTPlaqueTB test. Int J Tuberc Lung Dis 11: 904-908.

Lawn SD. 2012. Point-of-care detection of lipoarabinomannan (LAM) in urine for diagnosis of HIV-associated tuberculosis: A state of the art review. BMC Infect Dis 12: 103.

Lawn SD, Nicol MP. 2011. Xpert(R) MTB/RIF assay: Development, evaluation and implementation of a new rapid molecular diagnostic for tuberculosis and rifampicin resistance. Future Microbiol 6: 1067-1082.

Lawn SD, Wood R, Wilkinson RJ. 2011. Changing concepts of "latent tuberculosis infection" in patients living with HIV infection. Clin Dev Immunol 2011: 980594.

Lawn SD, Kerkhoff AD, Vogt M, Wood R. 2012a. Diagnostic accuracy of a low-cost, urine antigen, point-of-care screening assay for HIV-associated pulmonary tuberculosis before antiretroviral therapy: Descriptive study. Lancet Infect Dis 12: 201-209.

Lawn SD, Kerkhoff AD, Vogt M, Wood R. 2012b. Clinical significance of lipoarabinomannan detection in urine using a low-cost point-of-care diagnostic assay for HIV-associated tuberculosis. AIDS 26: 1635-1643.

Lawn SD, Mwaba P, Bates M, Piatek A, Alexander H, Marais BJ, Cuevas LE, McHugh TD, Zijenah L, Kapata N, et al. 2013. Advances in tuberculosis diagnostics: The Xpert MTB/RIF assay and future prospects for a point-ofcare test. Lancet Infect Dis 13: 349-361. 
S.D. Lawn

Lawn SD, Kerkhoff AD, Burton R, Meintjes G. 2014. Underestimation of the incremental diagnostic yield of HIVassociated tuberculosis in studies of the determine TBLAM Ag urine assay. AIDS 28: 1846-1848.

Lemaire JF, Casenghi M. 2010. New diagnostics for tuberculosis: Fulfilling patient needs first. J Int AIDS Soc 13: 40.

Ling DI, Zwerling AA, Pai M. 2008. GenoType MTBDR assays for the diagnosis of multidrug-resistant tuberculosis: A meta-analysis. Eur Respir J 32: 1165-1174.

MacPherson P, Houben RM, Glynn JR, Corbett EL, Kranzer K. 2014. Pre-treatment loss to follow-up in tuberculosis patients in low- and lower-middle-income countries and high-burden countries: A systematic review and metaanalysis. Bull World Health Organ 92: 126.

McNerney R, Daley P. 2011. Towards a point-of-care test for active tuberculosis: Obstacles and opportunities. Nat Rev Microbiol 9: 204-213.

Minion J, Leung E, Menzies D, Pai M. 2010. Microscopicobservation drug susceptibility and thin layer agar assays for the detection of drug resistant tuberculosis: A systematic review and meta-analysis. Lancet Infect Dis 10: 688698.

Minion J, Pai M, Ramsay A, Menzies D, Greenaway C. 2011a Comparison of LED and conventional fluorescence microscopy for detection of acid fast bacilli in a low-incidence setting. PLoS ONE 6: e22495.

Minion J, Leung E, Talbot E, Dheda K, Pai M, Menzies D. 2011b. Diagnosing tuberculosis with urine lipoarabinomannan: Systematic review and meta-analysis. Eur Respir J 38: $1398-1405$.

Mitarai S, Okumura M, Toyota E, Yoshiyama T, Aono A, Sejimo A, Azuma Y, Sugahara K, Nagasawa T, Nagayama $\mathrm{N}$, et al. 2011. Evaluation of a simple loop-mediated isothermal amplification test kit for the diagnosis of tuberculosis. Int J Tuberc Lung Dis 15: 1211-1217.

Moore DA, Evans CA, Gilman RH, Caviedes L, Coronel J, Vivar A, Sanchez E, Pinedo Y, Saravia JC, Salazar C, et al. 2006. Microscopic-observation drug-susceptibility assay for the diagnosis of TB. N Engl J Med 355: 1539-1550.

Morgan M, Kalantri S, Flores L, Pai M. 2005. A commercial line probe assay for the rapid detection of rifampicin resistance in Mycobacterium tuberculosis: A systematic review and meta-analysis. BMC Infect Dis 5: 62 .

Musa HR, Ambroggi M, Souto A, Angeby KA. 2005. Drug susceptibility testing of Mycobacterium tuberculosis by a nitrate reductase assay applied directly on microscopypositive sputum samples. J Clin Microbiol 43: 31593161.

Niemz A, Boyle DS. 2012. Nucleic acid testing for tuberculosis at the point-of-care in high-burden countries. Expert Rev Mol Diagn 12: 687-701.

Osman M, Simpson JA, Caldwell J, Bosman M, Nicol MP. 2014. GeneXpert MTB/RIF version G4 for identification of rifampin-resistant tuberculosis in a programmatic setting. J Clin Microbiol 52: 635-637.

Pai M, Zwerling A, Menzies D. 2008. Systematic review: T-cell-based assays for the diagnosis of latent tuberculosis infection: An update. Ann Intern Med 149: 177-184.

Palomino JC, Martin A, Portaels F. 2007. Rapid drug resistance detection in Mycobacterium tuberculosis: A review of colourimetric methods. Clin Microbiol Infect 13: 754762.

Perkins MD. 2009. New diagnostics for tuberculosis. In Tuberculosis-A comprehensive clinical reference (ed. Schaaf HS, Zumla A). Elsevier, London.

Peter J, Green C, Hoelscher M, Mwaba P, Zumla A, Dheda K. 2010. Urine for the diagnosis of tuberculosis: Current approaches, clinical applicability, and new developments. Curr Opin Pulm Med 16: 262-270.

Peter JG, Theron G, van Zyl-Smit R, Haripersad A, Mottay L, Kraus S, Binder A, Meldau R, Hardy A, Dheda K. 2012. Diagnostic accuracy of a urine LAM strip-test for TB detection in HIV-infected hospitalised patients. Eur Respir J 40: 1211-1240.

Phillips M, Basa-Dalay V, Blais J, Bothamley G, Chaturvedi A, Modi KD, Pandya M, Natividad MP, Patel U, Ramraje NN, et al. 2012. Point-of-care breath test for biomarkers of active pulmonary tuberculosis. Tuberculosis (Edinb) 92: $314-320$.

Robledo J, Mejia GI, Paniagua L, Martin A, Guzman A. 2008. Rapid detection of rifampicin and isoniazid resistance in Mycobacterium tuberculosis by the direct thinlayer agar method. Int J Tuberc Lung Dis 12: 1482-1484.

Steingart KR, Ng V, Henry M, Hopewell PC, Ramsay A, Cunningham J, Urbanczik R, Perkins MD, Aziz MA, Pai M. 2006a. Sputum processing methods to improve the sensitivity of smear microscopy for tuberculosis: A systematic review. Lancet Infect Dis 6: 664-674.

Steingart KR, Henry M, Ng V, Hopewell PC, Ramsay A, Cunningham J, Urbanczik R, Perkins M, Aziz MA, Pai M. 2006b. Fluorescence versus conventional sputum smear microscopy for tuberculosis: A systematic review. Lancet Infect Dis 6: $570-581$.

Steingart KR, Henry M, Laal S, Hopewell PC, Ramsay A, Menzies D, Cunningham J, Weldingh K, Pai M. 2007a. A systematic review of commercial serological antibody detection tests for the diagnosis of extrapulmonary tuberculosis. Thorax 62: 911-918.

Steingart KR, Henry M, Laal S, Hopewell PC, Ramsay A, Menzies D, Cunningham J, Weldingh K, Pai M. 2007b. Commercial serological antibody detection tests for the diagnosis of pulmonary tuberculosis: A systematic review. PLoS Med 4: e202.

Steingart KR, Dendukuri N, Henry M, Schiller I, Nahid P, Hopewell PC, Ramsay A, Pai M, Laal S. 2009. Performance of purified antigens for serodiagnosis of pulmonary tuberculosis: A meta-analysis. Clin Vaccine Immunol 16: $260-276$.

Steingart KR, Schiller I, Horne DJ, Pai M, Boehme CC, Dendukuri N. 2014. Xpert(R) MTB/RIF assay for pulmonary tuberculosis and rifampicin resistance in adults. Cochrane Database Syst Rev 1: CD009593.

Talbot E, Munseri P, Teixeira P, Matee M, Bakari M, Lahey T, von Reyn F. 2012. Test characteristics of urinary lipoarabinomannan and predictors of mortality among hospitalized HIV-infected tuberculosis suspects in Tanzania. PLOS ONE 7: e32876.

Trebucq A, Enarson DA, Chiang CY, Van DA, Harries AD, Boillot F, Detjen A, Fujiwara PI, Graham SM, Monedero I, et al. 2011. Xpert(R) MTB/RIF for national tuberculosis programmes in low-income countries: 
When, where and how? Int J Tuberc Lung Dis 15: $1567-$ 1572.

UNITAID. 2012. Tuberculosis diagnostic technology landscape. UNITAID, World Health Organization, Geneva, 2012. Accessible at: http://www.unitaid.eu/images/ marketdynamics/publications/UNITAID-TuberculosisLandscape_2012.pdf.

UNITAID. 2013. Tuberculosis diagnostics technology and market landscape. 2nd ed. UNITAID, World Health Organization, Geneva. Accessible at: http://www.unitaid.eu/images/marketdynamics/publications/TB-DxLandscape_1-Jul-2013.pdf.

Wallis RS, Kim P, Cole S, Hanna D, Andrade BB, Maeurer M, Schito M, Zumla A. 2013. Tuberculosis biomarkers discovery: Developments, needs, and challenges. Lancet Infect Dis 13: 362-372.

World Health Organization. 2007a. TB diagnostics and laboratory strengthening-WHO policy. Definition of a new sputum smear-positive TB cases. World Health Organization, Geneva. Accessible at: http://www.who.int/tb/ laboratory/policy_sputum_smearpositive_tb_case/en/ index.html.

World Health Organization. 2007b. TB diagnostics and laboratory strengthening-WHO policy. Reduction in the number of smears for the diagnosis of pulmonary TB. WHO, Geneva. Accessible at http://www.who.int/ tb/laboratory/policy_diagnosis_pulmonary_tb/en/index .html.

World Health Organization. 2007c. TB diagnostics and laboratory strengthening-WHO policy. The use of liquid medium for culture and DST. WHO, Geneva. Accessible at: http://www.who.int/tb/laboratory/policy_liquid_ medium_for_culture_dst/en/index.html.

World Health Organization. 2008. Molecular line probe assays for rapid screening of patients at risk of multidrug-resistant tuberculosis (MDR-TB). Policy statement June 2008. www.who.int/entity/tb/dots/laboratory/ lpa_policy.pdf.

World Health Organization 2010. Tuberculosis diagnostics automated DNA test. WHO endorsement and recommendations. World Health Organization, Geneva. Accessible at: http://www.who.int/tb/features_archive/ xpert_factsheet.pdf.

World Health Organization. 2011a. TB diagnostics and laboratory strengthening-WHO policy. Same-day diagnosis of tuberculosis by microscopy. WHO, Geneva. Accessible at: http://whqlibdoc.who.int/publications/2011/ 9789241501606_eng.pdf.

World Health Organization. 2011b. TB diagnostics and laboratory strengthening-WHO policy. Noncommerical culture and drug-susceptibility testing methods for screening patients at risk for multidrug-resistant tuberculosis. Accessible at: http://whqlibdoc.who.int/ publications/2011/9789241501620_eng.pdf.
Advances in Diagnostic Assays for Tuberculosis

World Health Organization. 2011c. TB diagnostics and laboratory strengthening-WHO policy. Fluorescent light-emitting diode (LED) microscopy for diagnosis of tuberculosis. Accessible at: http://whqlibdoc.who.int/ publications/2011/9789241501613_eng.pdf.

World Health Organization. 2011d. Commercial serodiagnostic tests of diagnosis of tuberculosis. WHO, Geneva. Accessible at: http://whqlibdoc.who.int/publications/ 2011/9789241502054_eng.pdf.

World Health Organization. 2011e. Use of tuberculosis interferon- $\gamma$ release assays (IGRAs) in low- and middleincome countries. WHO, Geneva. Accessible at: http:// whqlibdoc.who.int/publications/2011/9789241502672_ eng.pdf.

World Health Organization 2011f. Automated real-time nucleic acid amplification technology for rapid and simultaneous detection of tuberculosis and rifampicin resistance: Xpert MTB/RIF System Policy Statement. WHO, Geneva. Accessed on 28th June 2011 at: http://whqlib doc.who.int/publications/2011/9789241501545_eng.pdf.

World Health Organization. 2013a. Global tuberculosis report. World Health Organization, Geneva. Accessible at the following URL: http://apps.who.int/iris/bitstream/ 10665/91355/1/9789241564656_eng.pdf.

World Health Organization. 2013b. Xpert MTB/RIF for the diagnosis of pulmonary and extrapulmonary $\mathrm{TB}$ in adults and children: Policy update. WHO/HTM/ TB/2013.16. WHO, Geneva. Accessible at the following URL: http://apps.who.int/iris/bitstream/10665/ 112472/1/9789241506335_eng.pdf?ua=1.

World Health Organization. 2013c. The use of molecular line probe assay for the detection of resistance to second-line anti-tuberculosis drugs. Expert group meeting report, Geneva, February 2013. WHO, Geneva. Accessible at: http://apps.who.int/iris/bitstream/10665/78099/ 1/WHO_HTM_TB_2013.01.eng.pdf.

World Health Organization. 2013d. The use of a commercial loop-mediated isothermal amplification assay (TBLAMP) for the detection of tuberculosis. Expert group meeting report, Geneva, May 2013. WHO, Geneva. Accessible at: http://apps.who.int/iris/bitstream/10665/ 83142/1/WHO_HTM_TB_2013.05_eng.pdf.

Yerushalmy J. 1947. Statistical problems in assessing methods of medical diagnosis, with special reference to X-ray techniques. Public Health Rep 62: 1432-1449.

Young DB, Gideon HP, Wilkinson RJ. 2009. Eliminating latent tuberculosis. Trends Microbiol 17: 183-188.

Yu X, Prados-Rosales R, Jenny-Avital ER, Sosa K, Casadevall A, Achkar JM. 2012. Comparative evaluation of profiles of antibodies to mycobacterial capsular polysaccharides in tuberculosis patients and controls stratified by HIV status. Clin Vaccine Immunol 19: 198-208. 


\section{$\$ \mathrm{CSH} \&$ Cold Spring Harbor

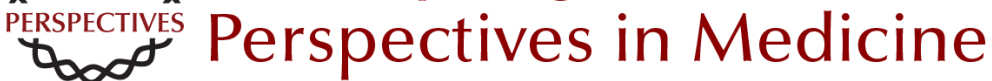

\section{Advances in Diagnostic Assays for Tuberculosis}

Stephen D. Lawn

Cold Spring Harb Perspect Med 2015; doi: 10.1101/cshperspect.a017806 originally published online August 7, 2015

\section{Subject Collection Tuberculosis}

Transmission and Institutional Infection Control of Tuberculosis Edward A. Nardell

Innate and Adaptive Cellular Immune Responses

to Mycobacterium tuberculosis Infection Katrin D. Mayer-Barber and Daniel L. Barber

Tuberculosis Comorbidity with Communicable and Noncommunicable Diseases

Matthew Bates, Ben J. Marais and Alimuddin Zumla

Host-Directed Therapies for Tuberculosis David M. Tobin

Immunity and Immunopathology in the Tuberculous Granuloma Antonio J. Pagán and Lalita Ramakrishnan

Tuberculosis Drug Development: History and Evolution of the Mechanism-Based Paradigm? Sumit Chakraborty and Kyu Y. Rhee

Genetic Approaches to Facilitate Antibacterial Drug Development Dirk Schnappinger

The Tuberculosis Drug Discovery and Development Pipeline and Emerging Drug Targets Khisimuzi Mdluli, Takushi Kaneko and Anna Upton
Clinical Aspects of Adult Tuberculosis Robert Loddenkemper, Marc Lipman and Alimuddin Zumla

Advances in Diagnostic Assays for Tuberculosis Stephen D. Lawn

Diagnosis and Management of Latent

Tuberculosis Infection

Laura Muñoz, Helen R. Stagg and Ibrahim Abubakar

Mycobacterial Growth Iria Uhía, Kerstin J. Williams, Vahid Shahrezaei, et al.

Multidrug-Resistant Tuberculosis and Extensively

Drug-Resistant Tuberculosis Kwonjune J. Seung, Salmaan Keshavjee and Michael L. Rich

The Mycobacterial Cell Wall--Peptidoglycan and

Arabinogalactan Luke J. Alderwick, James Harrison, Georgina S. Lloyd, et al.

Tuberculosis and HIV Coinfection Judith Bruchfeld, Margarida Correia-Neves and Gunilla Källenius

Imaging in Tuberculosis Jamshed B. Bomanji, Narainder Gupta, Parveen Gulati, et al.

For additional articles in this collection, see http://perspectivesinmedicine.cshlp.org/cgi/collection/ 